\title{
Specifying Complex Systems with Bayesian Programming. An Alife Application ${ }^{\star}$
}

\author{
Fidel Aznar, Mar Pujol, and Ramón Rizo \\ Department of Computer Science and Artificial Intelligence, \\ University of Alicante \\ \{fidel, mar, rizo\}@dccia.ua.es
}

\begin{abstract}
One of the most important application areas of Artificial Life is the simulation of complex processes. This paper shows how to use Bayesian Programming to model and simulate an artificial life problem: that of a worm trying to live in a world full of poison. Any model of a real phenomenon is incomplete because there will always exist unknown, hidden variables that influence the phenomenon. To solve this problem we apply a new formalism, Bayesian programming. The proposed worm model has been used to train a population of worms using genetic algorithms. We will see the advantages of our method compared with a classical approach. Finally, we discuss the emergent behaviour patterns we observed in some of the worms and conclude by explaining the advantages of the applied method. It is this characteristic (the emergent behaviour) which makes Artificial Life particularly appropriate for the study and simulation of complex systems for which detailed analysis, using traditional methods, is practically non-viable.
\end{abstract}

Keywords: Bayesian Programming, Complex Systems Modeling, Artificial Life Formalization Model.

\section{Introduction}

Initially, Artificial Life was defined as a broad field of work in which attempts are made to simulate or recreate one or more natural processes using artificial methods. Nevertheless, applications in this field have quickly exceeded purely biological applications. The immediate applications of Artificial Life are in the simulation of complex processes, chemical synthesis, multivariate phenomena, etc. Very complex global behaviour patterns can be observed, initiated by simple local behaviour. It is this characteristic (sometimes called emergent behaviour) which makes Artificial Life particularly appropriate for the study and simulation of complex systems for which detailed analysis, using traditional methods, is practically non-viable. Nevertheless, it is necessary to bear in mind that any model of a real phenomenon will always be incomplete due to the permanent

\footnotetext{
* This work has been financed by the Generalitat Valenciana project GV04B685.
} 
existence of unknown, hidden variables that will influence the phenomenon. The effect of these variables is malicious since they will cause the model and the phenomenon to have different behavioural patterns. In this way both artificial systems and natural systems have to solve a common problem: how each individual within the system uses an incomplete model of the environment to perceive, infer, decide and act in an efficient way.

Reasoning with incomplete information continues to be a challenge for artificial systems. Probabilistic inference and learning try to solve this problem using a formal base. A new formalism, the Bayesian programming (BP) [1], based on the principle of the Bayesian theory of probability, has been successfully used in autonomous robot programming. Bayesian programming is proposed as a solution when dealing with problems relating to uncertainty or incompleteness. Certain parallelisms exist between this kind of programming and the structure of living organisms as shown in a theoretical way in 2 .

We will see a simple example of how to apply BP formalism to a specific artificial life problem. We will define a virtual world, divided into cells, some of which contain poison. In this world lives a worm with only one purpose, to grow indefinitely. In order to grow the worm must move through a certain number of non poisonous cells in its world. If the worm moves into a poisonous cell then it will die. The worm has a limited vision of the world, provided by its sensorial organs, found in its head. These sensors allow the worm to see no further than the adjacent cell. We believe that this is one of the first approaches that uses Bayesian programming for the formalization of an artificial life problem as we haven't found any evidence of it's application in this field.

\section{$2 \quad$ Bayesian Programming}

Using incomplete information for reasoning continues to be a challenge for artificial systems. Probabilistic inference and learning try to solve this problem using a formal base. Bayesian programming has been used successfully in autonomous robot programming [2], [1], 3], 4], [5]. Using this formalism we employ incompleteness explicitly in the model and then, the model's uncertainty chosen by the programmer, are defined explicitly too.

A Bayesian program is defined as a mean of specifying a family of probability distributions. There are two constituent components of a Bayesian program. The first is a declarative component where the user defines a description. The purpose of a description is to specify a method to compute a joint distribution. The second component is of a procedural nature and consists of using a previously defined description with a question (normally computing a probability distribution of the form $P($ Searched $\mid$ Known $))$. Answering this question consists in deciding a value for the variable Searched according to $P($ Searched $\mid K$ nown $)$ using the Bayesian inference rule:

$$
\begin{aligned}
& P(\text { Searched } \mid \text { Known } \otimes \delta \otimes \pi)= \\
& \frac{1}{\Sigma} \times \sum_{\text {Unknown }} P(\text { Searched } \otimes \text { Unknown } \otimes \text { Known } \mid \delta \otimes \pi)
\end{aligned}
$$


It is well known that a general Bayesian inference is a very difficult problem, which may be practically intractable. However, for specific problems, it is assumed that the programmer would implement an inference engine in an efficient manner. More details about BP can be found in [1, 2].

\section{$3 \quad$ Specifying the Problem Using Bayesian Programming}

We commented above on the existence of a world, composed of $n \times m$ cells, where each cell $C_{i j}$ could be in any one of four different states: empty, containing poison, part of the wall which surrounds this artificial world or it could be hidden from view beneath the worm's tail. In this way a cell $C_{i j}=\{\emptyset, V, M, L\}$. The wall configuration is uniform for each generated world, however, in contrast, the distribution of poison is random and varies from world to world. Initially, we asume the amount of poisonous cells to be between $5 \%-10 \%$ of the total. Within each world lives only a single worm which only objective is to move and to grow. A worm grows and increases its length by one unit every time it moves through $d$ cells inside its world. If the worm moves to a cell that is not empty then it will die. The only information about the world available to the worm is provided by its sensors, located in its head (see figure 1a). A sensor is only able to see the state of cells adjacent to and in front of the worm's head, no further.

We assume that each worm has a certain knowledge represented as states. In this way each worm can stay in one state $E_{t}$ given a reading and a previous state. Furthermore, a worm could obtain a reading of the world $L_{t}$ represented as a binary triplet which specifies if the cell in the position of its components is occupied '1' or not '0'. Finally, a worm could execute three actions. Go straight ahead, turn left or turn right $A_{t}=\{u, l, r\}$ the actions will be guided only by a reading and the actual state of the worm. Once the action $A_{t}$ has been executed the worm can change to a new state $E_{t+1}$.

The first part of a Bayesian program is to define the pertinent variables of the problem. To develop a movement in the world, the worm only needs to know the reading $L_{t}$ of it's sensor and the actual state $E_{t}$, in addition to the set of actions $A$ it could develop in the world. As we commented previously, an action $A_{t}$ must be followed by an instant change in state $t+1$. In this way we define the following variables for each instant $t$ :

$$
\begin{array}{r}
L_{t}=\{000,001,010, \ldots, 111\},\left\lfloor L_{t}\right\rfloor=8 \\
E_{t}=\{0,1,2, \ldots, k\},\left\lfloor E_{t}\right\rfloor=k+1 \\
A_{t}=\{u, l, r\},\lfloor A\rfloor=3
\end{array}
$$

The second part of a BP is to define a decomposition of the joint probability distribution $P\left(L_{t} \otimes E_{t-1} \otimes E_{t} \otimes A \mid \pi_{W}\right)$ as a product of simpler terms. This distribution is conditioned by the previous knowledge $\pi_{w}$ we are defining. 


$$
\begin{array}{r}
P\left(L_{t} \otimes E_{t-1} \otimes E_{t} \otimes A_{t} \mid \pi_{W}\right)=P\left(L_{t} \mid \pi_{W}\right) \times P\left(E_{t-1} \mid L_{t} \otimes \pi_{W}\right) \times \\
\times P\left(E_{t} \mid E_{t-1} \otimes L_{t} \otimes \pi_{W}\right) \times P\left(A_{t} \mid E_{t} \otimes E_{t-1} \otimes L_{t} \otimes \pi_{W}\right)= \\
=P\left(L_{t} \mid \pi_{W}\right) \times P\left(E_{t-1} \mid L_{t} \otimes \pi_{W}\right) \times \\
\times P\left(E_{t} \mid E_{t-1} \otimes L_{t} \otimes \pi_{W}\right) \times P\left(A_{t} \mid E_{t} \otimes L_{t} \otimes \pi_{W}\right)
\end{array}
$$

The second equality is deduced from the fact that an action only depends on the actual state and the reading taken.

Next, in order to be able to solve the joint distribution, we need to assign parametrical forms to each term appearing in the decomposition:

$$
\begin{array}{r}
P\left(L_{t} \mid \pi_{W}\right) \equiv \text { Uniform } \\
P\left(E_{t-1} \mid L_{t} \otimes \pi_{W}\right) \equiv \text { Uniform } \\
P\left(E_{t} \mid E_{t-1} \otimes L_{t} \otimes \pi_{W}\right) \equiv G\left(\mu\left(E_{t-1}, L_{t}\right), \sigma\left(E_{t-1}, L_{t}\right)\right) \\
P\left(A_{t} \mid E_{t} \otimes L_{t} \otimes \pi_{W}\right) \equiv G\left(\mu\left(E_{t}, L_{t}\right), \sigma\left(E_{t}, L_{t}\right)\right)
\end{array}
$$

We assume that the probability of a reading is uniform because we have no prior information about the distribution of the world. In the same way we consider that all possible worm states can be reached with the same probability. Give a state $E_{t-1}$ and a lecture $L_{t}$ we believe that only one state $E_{t}$ would be preferred. In this way the distribution $P\left(E_{t} \mid E_{t-1} \otimes L_{t} \otimes \pi_{W}\right)$ is unimodal. However, depending on the situation, the decision to be made may be more or less certain. This behaviour is resumed by assigning a Gaussian parametrical form to $P\left(E_{t} \mid E_{t-1} \otimes L_{t} \otimes \pi_{W}\right)$. In the same way, given a state and a reading we suppose that an action with more or less intensity would be prepared.

We show a set of free parameters which define the way the worm moves. These free parameters (that must be identified), derived from the parametrical form (means and standard deviations of all the Gaussians $\left\lfloor E_{t-1}\right\rfloor \times\left\lfloor L_{t}\right\rfloor$ and $\left\lfloor E_{t}\right\rfloor \times\left\lfloor L_{t}\right\rfloor$ ), would be the ones to be learned.

Finally we specify the steps that the worm needs to move (using the joint distribution): to obtain a reading $L_{t}$ from the worm's sensors, to answer the question $\operatorname{Draw}\left(P\left(A_{t} \mid E_{t} \otimes L_{t} \otimes \pi_{W}\right)\right)$, then the worm will execute the movement command $A$ and will answer the question $\operatorname{Draw}\left(P\left(E_{t+1} \mid E_{t} \otimes L_{T} \otimes \pi_{W}\right)\right)$, finally the worm will change to the state $E_{t+1}$.

\section{Genetic Algorithms}

Genetic algorithms (GA) are a global search technique which mimic aspects of biological evolution. We initially assume that the worm's parameters are generated randomly. The worm only has previous knowledge provided by its knowledge decomposition. The learning process would be produced generation after generation, where the longest living worms in the world would be those most enabled and adapted to reproduce and to maintain their intelligence. Next we will describe the main parts of our genetic algorithm.

Chromosome Codification. A chromosome is represented using two tables. The first one is formed by $2 \cdot k \cdot 8$ components specifying the Gaussians $\left\lfloor E_{t-1}\right\rfloor \times$ $\left\lfloor L_{t}\right\rfloor$ which represent $P\left(E_{t} \mid E_{t-1} \otimes L_{t} \otimes \pi_{W}\right)$. The second table is formed by the 
same component numbers specifying the Gaussians $\left\lfloor E_{t}\right\rfloor \times\left\lfloor L_{t}\right\rfloor$ which represent $P\left(A_{t} \mid E_{t} \otimes L_{t} \otimes \pi_{W}\right)$. In this way, each chromosome contains $32 \cdot k$ gens. In the described experiments, the initial chromosome population is obtained by randomly initializing the Gaussian parameters.

Fitness Function. We want to reward the worms that live the longest time in the world. In this way we describe the fitness function as the number of iterations that a worm lives in a randomized generated world. In order to avoid the situation where a simple world produces an overvalued worm, we generate $w$ random worlds to evaluate each worm's fitness. All worlds are the same size and have the same wall disposition, only the quantity and position of poisonous cells varies, being selected randomly and comprising between $5 \%$ and $10 \%$ of the total cells.

\section{Selection, Crossover an Mutation Operators.}

- Selection operator. We used a stochastic remainder sampling selector (SRS) with a two-staged selection procedure. In addition we use elitism (the best individual from each generation is carried over to the next generation).

- Crossover operator. We use an asexual two-point crossover operator. In this way the mother genes will be selected until the crossover point where the father genes will be copied. This process will be done for the two tables (see figure 1b) that describe the chromosome.

- Mutation operator. We define an incremental mutation operator for states, in this way given a gene $x$ we define a mutation as: $x \in[0, k], \operatorname{mut}(x)=$ $x+1 M O D k$. Suppose we have four states, and that $q_{2}=3$, if we mutate this element we will obtain $q_{2}=3+1 M O D 4=0$. A random mutation scheme is used to choose the directions for the worm to take. A new direction is generated randomly and then substitutes the original gene.

\subsection{Used Parameters}

Using the operators presented in the previous section we obtain an evolutive learning process for a worm. Developing empirical tests we arrive at the conclusion that a number of states $(k)$ greater than five complicates the learning process of the worm and does not improve the movements made by the worm. For this reason, in the rest of the experiments, we use a fixed number of states equal to five.In addition, for the remainder of tests we use $d=5$ (for each five cells the worm moves through it will increase it's size by one unit) and $w=6$ (six random worlds will be generated in order to evaluate each worm).

\subsection{Worms Evolution}

In order to obtain the best individual we use 100 executions for each state with a population of 250 individuals and 500 generations using the operators specified in the previous section. In figure 1c an example is shown of the executions showing the fitness of the worst and best performing individual as well as the average results obtained, illustrating the algorithm convergence. For each algorithm execution the evaluation took about 2 minutes using a Pentium IV running at $2 \mathrm{Ghz}$. 

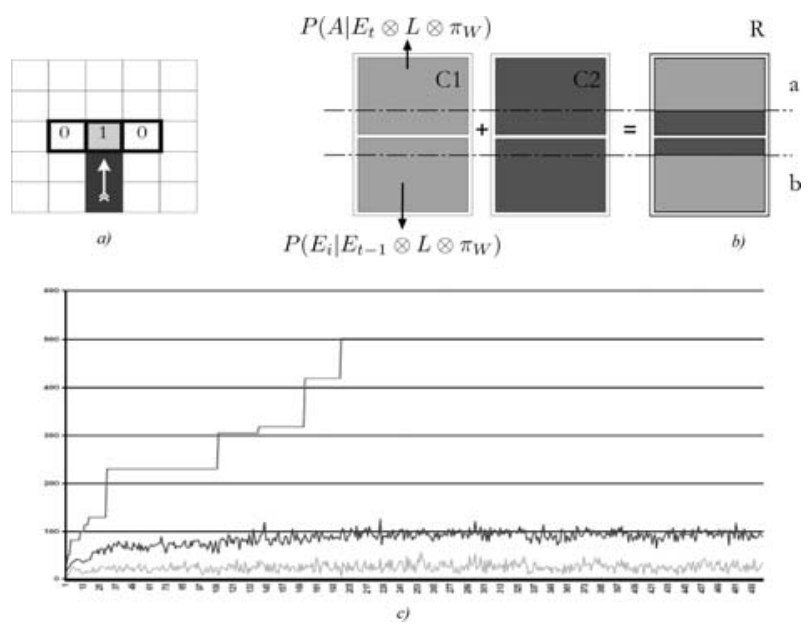

Fig. 1. a) Worm's vision relating to its head and the direction it has in the world. b) Asexual two point crossover operator for the worm's chromosome. c) Evolution of the worst (bottom line), the average (in the middle) and the best performing individual (top). The $y$ axis represents the worm's fitness and the $x$ axis the actual generation. Until the first 50 iterations an improvement is produced in the medium and the worst individual. Then the graph tends to oscillate although a slight increase is produced (because the best case increases and maintains it's level through elitism)

\section{Survival Behaviours and Experimentation}

In this section we will analyze some characteristics and emergent behaviours that were observed in the worms. Readers of this paper are invited to test our simulator at the following address http://www.dccia.ua.es/ fidel/worm.zip. Using Bayesian Programming the worm's previous knowledge is defined and mechanisms are given to provide new knowledge to the worm. This data is represented using two sets of discrete Gaussians which were learned using genetic algorithms. However, we should remember that to get the information of the learned distributions we use the Draw function which randomly extracts a value for the distribution. In this way we obtain a non-deterministic behaviour, which is more adaptable to variations in complex worlds. After training the worm population we simulate, in a graphical way, the best individual found. It is curious to see different behaviour patterns, which provide more survival opportunities. Some of these patterns even seem to imitate natural behaviour developed in some animals.

One of the most common patterns is to follow the edge of the world while no poison is found near it (see figure 2a). This is a good way to move if the proportion of poison is low near the edges and configurations don't exist that trap the worm between the perimeters and the poison. Another curious behaviour is the development of a zigzag movement emulating the way some snakes move (see figure 2b) so reducing the area that the worm occupies in the world. In addition it is quite common for the worm to move up and down like a ping-pong ball 


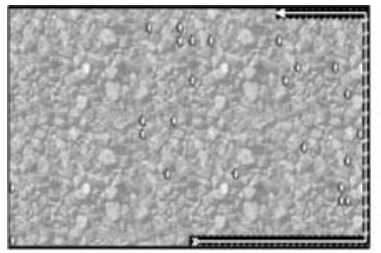

d)

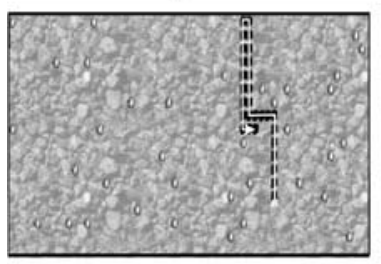

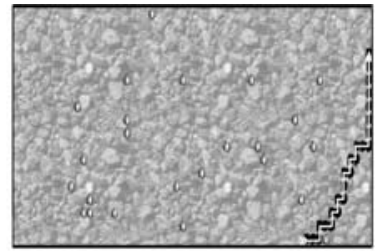

b)

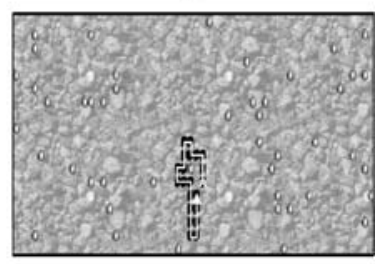

d)

Fig. 2. Different behavior patterns. a) Follow the edge of the world. $b$ ) Zigzag movement. c) Ping-pong behaviour. d) In the movement the worm seems to follow its tail. The arrow points to the next worm displacement

(see figure 2:). Finally, we underline the movement of some worms which seem to move as if trying to reach their tails, so forming a spiral (see figure $2 \mathrm{~d}$ ). The behaviour described above (and some others) are repeated and combined with the obtained worms. These behaviour are not programmed implicitly, they have been obtained using the proposed brain model and selected using an evolving process in a population.

\subsection{Comparing Our Model with a Clasical One}

We can see some advantages of our method if we compare it to a more classical model approach, the finite states machine (FSM) [6]. This approach has various drawbacks. First, it assumes a perfect world model, which is false. It is necessary to know that any model of a real phenomenon is incomplete because there will always exist non-considered, hidden variables, that will influence the phenomenon. The effect of these variables is malicious since they will cause the model and the phenomenon to show different behavioural patterns. Second, a FSM develop a deterministic behaviour therefore in certain world configurations it will fail. On the other hand, a Bayesian model has not a deterministic behaviour and two different executions in the same world may have different results, which provide greater adaptability to changes in the environment configuration.

\section{Conclusions}

In this paper we have seen an application of Bayesian programming in an artificial life system. The formalism of the artificial life models is a continuous field of investigation because of the complexity of the systems we work with 
[7, 8]. In addition, we have the added difficulty of working with uncertainty and include it into the model we want to use. The Bayesian programming brings up a formalism where implicitly, using probabilities, we work with the uncertainly.

In a world with randomly distributed poison lives a worm, which main purpose is to grow up. We propose a formalization of the virtual worm using a decomposition in terms of a joint probability distribution of their knowledge. In this way, applying the Bayesian Programming we obtain a versatile behaviour adaptable to changes and what is more a mathematical description of the probabilistic environment model. We have seen some advantages of our method comparing it to a more classical model approach, the finite states machine (FSM 6]) (see section 5.1).The learning process, given a worm population, has been developed with evolving techniques, using genetic algorithms. The principal reason for using GA was because they are a global search technique which mimic aspects of biological evolution even though other search techniques could be picked to select the worms. Each used chromosome is the codification of the two distributions obtained with the previous Bayesian formalism. Satisfactory results were obtained that prove the validity of the proposed model. Relatively complex and elaborate behavioural patterns were observed in the movements of the most highly adapted worms. These behaviour patterns were not implicitly programmed but were obtained in an emergent way using the proposed model.

Bayesian programming is, therefore, a promising way to formalize both artificial and natural system models. In this example, we have seen how this paradigm can be adapted to a simple, artificial life problem. Future studies will try to model different artificial life systems using this new formalism.

\section{References}

1. Lebeltel, O., Bessire, P., Diard, J., Mazer, E.: Bayesian robots programming. Autonomous Robots 16 (2004) 49-79

2. Bessire, P., Group, I.R.: Survei:probabilistic methodology and tecniques for artefact conception and development. INRIA (2003)

3. Koike, C., Pradalier, C., Bessiere, P., Mazer, E.: Proscriptive bayesian programming application for collision avoidance. Proc. of the IEEE-RSJ Int. Conf. on Intelligent Robots and Systems (IROS); Las Vegas, USA (2003)

4. C. Cou, Th. Fraichard, P.B., Mazer, E.: Using bayesian programming for multisensor data fusion in automotive applications. IEEE Intelligent Vehicle Symposium (2002)

5. Bellot, D., Siegwart, R., Bessire, P., Cou, C., Tapus, A., Diard, J.: Bayesian reasoning for real world robotics: Basics, scaling and examples. Book Chapter in LNCS/LNAI, http://128.32.135.2/users/bellot/files/ David_Bellot_LNCS_LNAI.pdf (2004)

6. Dysband, E.: Game Programming Gems. A finite-state machine class (237-248). Charles River Media (2000) 
7. Agre, P., Horswill, I.: Lifeworld analysis. Journal of Artificial Intelligence Research 6 (1997) 111-145

8. Rasmussen, S., L. Barrett, C.: Elements of a theory of simulation. ECAL1995 (Advances in Artificial Life, Third European Conference on Artificial Life, Granada, Spain). (1995) 515-529 